\title{
CaMKK2-Mediated Effects on Mechanically Induced Bone Formation in Male and Female
} Mice

\author{
Margaret Bello ${ }^{1}$, Adam Warrick ${ }^{1}$, Brett Mattingly ${ }^{1}$, Justin Williams ${ }^{1}$, Uma Sankar ${ }^{1}$ \\ ${ }^{1}$ Department of Anatomy, Cell Biology and Physiology, Indiana University School of Medicine, \\ Indianapolis, IN.
}

Background and Hypothesis: Ca2+/calmo-dulin-dependent protein kinase kinase 2 (CaMKK2) is a serine-threonine protein kinase that plays a significant role in both anabolic and catabolic pathways of bone remodeling. Mechanical loading of bone translates an external force into both biochemical and structural changes. It has been shown that deletion or inhibition of CaMKK2 results in increased bone density in male and female mice. We hypothesize that the lack of CaMKK2 in bone cells will result in loading-induced bone mass accrual with no difference between male and female mice.

Experimental Design or Project Methods: The right tibia of anesthetized 16-week-old wildtype (WT) and CaMKK2 knockout (KO) mice were loaded at $2 \mathrm{~Hz}$ for 220 cycles and with peak forces specific to both sex and genotype. Loading was accomplished using an electro actuator (Bose ElectroForce 3200; EnduraTEC, Minnetonka, MN, USA). This was repeated 3, 5, 8 and 10 days after initial loading. The non-loaded left tibia served as an internal control. Calcein and alizarin red were administered intraperitoneally on days 9 and 16, respectively to metabolically label newly formed bone. Nineteen days after initial loading, mice were sacrificed. Blood and long bones of the lower limbs were collected for analysis.

Results: Using microcomputer tomography; dynamic histomorphometry; histology, immunohistochemistry, enzyme-linked immunosorbent assay and real-time reverse transcription-polymerase chain reaction, we will assess bone volume, bone formation rate, and underlying mechanisms at the cellular and molecular level. These data are forthcoming.

Conclusion and Potential Impact: With expanded knowledge on how bone growth is augmented, clinical outcomes related to osteoporosis and fracture healing, for example, may be improved. This may be accomplished through novel therapy related to these pathways that increases bone density or decreases fracture healing time. 